\title{
Schweigepflicht und Datenschutz bei der Abrechnung ärztlicher Leistungen
}

Viele Ärzte nutzen für privatärztliche Abrechnungen auf der Basis der Gebührenordnung für Ärzte (GOÄ) das Angebot von Abrechnungsstellen. Dies gilt sowohl für niedergelassene Ärzte als auch für liquidationsberechtigte Krankenhausärzte. Für die Abrechnung über eine Abrechnungsstelle ist dabei eine Abtretung der Rechnungsforderung gegenüber dem Patienten durch den Arzt an die Abrechnungsstelle notwendig. Gerade diese Abtretung birgt jedoch rechtliche Schwierigkeiten. Die Problematik reicht dabei von Datenschutzfragen bis hin zum klassischen Zivil- und Strafrecht.

\section{Mögliche Verletzung der Schwei- gepflicht \\ $\nabla$}

§ 203 Abs. 1 Nr. 1 StGB sanktioniert die Verletzung der ärztlichen Schweigepflicht mit Freiheitsstrafe bis zu einem Jahr oder Geldstrafe. Diese ärztliche Schweigepflicht wird nach der ständigen Rechtsprechung des BGH zwingend verletzt, wenn Patientendaten an eine Abrechnungsstelle weitergegeben werden. Dies liegt nach Ansicht des Gerichts darin begründet, dass der Arzt bei der Abtretung der Abrechnungsstelle gemäß § 402 BGB alle zur Geldendmachung der Forderung notwendigen Informationen und Auskünfte geben und entsprechende Beweismitteln aushändigen muss, was zwingend ein Verstoß gegen die ärztliche Schweigepflicht gemäß § 203 Abs. 1 Nr. 1 StGB darstelle. Eine Ausnahme gilt nur dann, wenn z. B. die Abrechnung wahlärztlicher Leistungen durch eine Abteilung innerhalb des Krankenhauses erfolgt (LG Bonn, Urt. v. 15.02.1995; Az.: 5 S 210/94). Dagegen handelt es sich bei einer Abrechnungsstelle um einen externen Dritten (LG Bonn, a. a. O.).

In der juristischen Literatur wird intensiv diskutiert, ob es sich bei Abrechnungsstellen um Gehilfen im Sinne des $§ 203$ Abs. 3 S. 2 StGB handeln könnte, die gleichsam in die Schweigepflicht des Arztes einbezogen werden. Abgesehen davon, dass diese schlecht mit den damit ursprünglich er- fassten Personengruppen wie z. B. Medizinischen Fachangestellten oder Rechtsanwaltsfachangestellten verglichen werden können, so wird dadurch der Kreis der von möglichen strafrechtlichen Sanktionen betroffenen Personen unter Verstoß gegen das strenge strafrechtliche Analogieverbot über Gebühr ausgedehnt (Bucher, MedR 2013, S. 337 ff., 339). Weiterhin ist es durchaus fraglich, ob eine vertragliche Vereinbarung so gestaltet werden kann, dass der Arzt auch gegenüber der Abrechnungsstelle in dieser Weise „Herr des Verfahrens“ ist, da es ja sein eigentliches Ziel ist, durch die Abrechnungsstelle entlastet zu werden.

\section{Die rechtfertigende Einwilligung des Patienten \\ $\nabla$}

Diese mögliche Verletzung des $§ 203$ Abs. $1 \mathrm{Nr} .1$ StGB kann jedoch durch eine Einwilligung des Patienten gerechtfertigt werden. Der BGH (Urt. v. 10.10.2013; Az.: III ZR 325/12) stellt dabei die Voraussetzungen auf, „dass der Erklärende eine im Wesentlichen zutreffende Vorstellung davon hat, worin er einwilligt, und die Bedeutung und Tragweite seiner Entscheidung zu überblicken vermag." Das bedeutet nach dem BGH, dass der Patient über Anlass und Zielsetzung der Schweigepflichtentbindung unterrichtet werden muss, sowie über die Identität der Personen, auf die sich die Schweigepflichtentbindung bezieht. Ebenso muss er wissen, wie und in welchem Umfang die Einschaltung Dritter stattfindet.

\section{Datenschutzrechtliche Problema- tik \\ $\nabla$}

Auch das Datenschutzrecht muss beachtet werden. $\S 11$ BDSG regelt dabei den Fall, dass personenbezogene Daten im Auftrag durch andere Stellen erhoben, verarbeitet oder genutzt werden. Diese Auftragsdatenverarbeitung unter Kontrolle und auf Weisung des Auftraggebers ist zwar privilegiert, aber dann nicht mehr anwendbar, wenn die Forderung nicht nur zur Erstel-

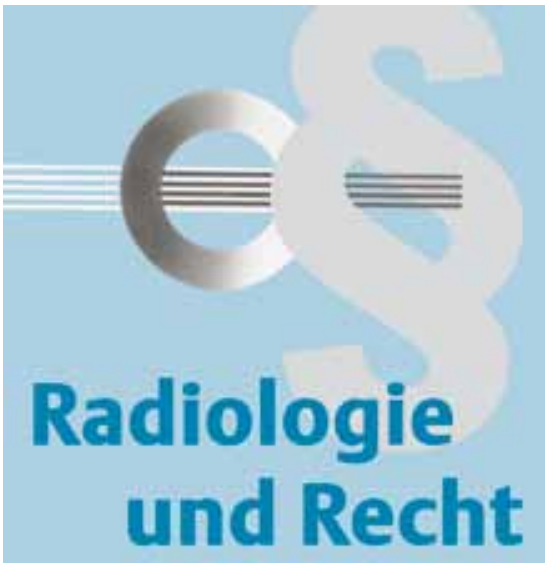

lung oder Versendung von Rechnungen, sondern auch für andere eigenständigere Zwecke, zum Beispiel zum Inkasso und einer etwaigen gerichtlichen Durchsetzung abgetreten wird (BGH, Urt. v. 10.07.1991; Az.: VIII ZR 296/90). Da dies in der Praxis oft gewollt sein wird, muss dafür eine datenschutzrechtliche Einwilligung des Patienten eingeholt werden. Diese muss gemäß § 4a Abs. 1 S. 3 BDSG schriftlich erfolgen. Da es sich um Gesundheitsdaten gemäß § 3 Abs. 9 BDSG handelt, muss sich die Einwilligung gemäß § 4a Abs. 3 BDSG explizit auf diese Daten beziehen.

\section{Der aktuelle Fall des BGH $\nabla$}

Aus diesen strafrechtlichen und datenschutzrechtlichen Tatbeständen können aber auch Konsequenzen für das Zivilrecht folgen. Über eine mögliche Unwirksamkeit einer Einwilligung in die Datenweitergabe und eine daraus folgende Nichtigkeit der gesamten Abtretungskonstruktion musste kürzlich der BGH entscheiden (Urt. v. 10. 10. 2013, Az.: III ZR $325 / 12)$.

Dem lag folgender Sachverhalt zu Grunde: Eine Abrechnungsstelle hatte nach Abtretung durch den Zahnarzt dem Patienten die Behandlungskosten in Rechnung gestellt. Der Patient hatte vorher folgende Erklärung unterschrieben, die, wie oben beschrieben, zwischen der Einwilligung zur Abtretung und der datenschutzrechtlichen Einwilligung unterschied:

\section{„Einwilligung zur Abtretung}

- Ich erkläre mich damit einverstanden, dass der umseitig genannte Zahnarzt zum Zweck der Erstellung der Rechnung sowie zur Einziehung und der gegebenenfalls gerichtlichen Durchsetzung der Forderung alle hierzu notwendigen Un- 
terlagen, insbesondere meinen Namen, Anschrift, Geburtsdatum, Leistungsziffern, Rechnungsbetrag, Behandlungsdokumentation, Laborrechnungen, Formulare etc. an die [Z-Abrechnungsgesellschaft] weitergibt.

- Insoweit entbinde ich den Zahnarzt ausdrücklich von seiner ärztlichen Schweigepflicht und stimme ausdrücklich $z u$, dass der Zahnarzt die sich aus der Behandlung ergebende Forderung an die [Z-Abrechnungsgesellschaft] und diese gegebenenfalls an das refinanzierende Institut - D.-Bank e. G., D. - abtritt.

- Ich bin mir bewusst, dass nach der Abtretung der Honorarforderung mir gegenüber die [Z-Abrechnungsgesellschaft] als Forderungsinhaberin auftritt und deshalb Einwände gegen die Forderung - auch soweit sie sich aus der Behandlung und der Krankengeschichte ergeben - im Streitfall gegenüber der [Z-Abrechnungsgesellschaft] $z u$ erheben und geltend zu machen sich und der mich behandelnde Zahnarzt als Zeuge vernommen werden kann.

\section{Einwilligung nach Datenschutzgesetz}

Ich bin gleichfalls damit einverstanden, dass meine persönlichen Daten und meine Behandlungsdaten von dem Zahnarzt und der [Z-Abrechnungsgesellschaft] gegebenenfalls elektronisch- erhoben, gespeichert, verarbeitet, genutzt und übermittelt werden zum Zweck der Erstellung der Honorarrechnung sowie der Einziehung und gegebenenfalls gerichtlichen Durchsetzung der Forderung. “

Im Anschluss fand tatsächlich nur die Abtretung durch den Zahnarzt an die Abrechnungsstelle statt, jedoch keine weitere Abtretung an die Bank. Der Patient weigerte sich aus verschiedenen Gründen, der Honorarforderung nachzukommen. Er bestritt nicht nur die Höhe der Rechnung an sich und behauptete, bei der Vergütungsvereinbarung geschäftsunfähig gewesen zu sein, sondern trug auch vor, dass nur eine unzureichende Aufklärung über die entsprechenden Abtretungen stattgefunden habe. Im Laufe des gerichtlichen Instanzenzuges vertrat in der zweiten Instanz das Oberlandesgericht (OLG) Braunschweig (Urt. v. 13.09.2012; Az.: 1 U 31/11) die Auffassung, die gesamte Abtretungskonstruktion sei unwirksam, da zwar der Umfang der Datenweitergabe und der Entbindung von der Schweigepflicht hinsichtlich der Abrechnungsstelle detailliert aufgeführt worden sei, nicht je- doch hinsichtlich der refinanzierenden Bank. Damit verstoße die Abtretungskonstruktion gegen die ärztliche Schweigepflicht gemäß § 203 Abs. 1 Nr. 1 StGB und sei damit aufgrund eines Verstoßes gegen ein gesetzliches Verbot gemäß § 134 BGB insgesamt nach $\S 139$ BGB nichtig. Diese Nichtigkeit erstrecke sich, so das OLG Braunschweig, aufgrund des engen $\mathrm{Zu}$ sammenhangs beider Abtretungen auch auf die Abtretung gegenüber der Abrechnungsstelle.

\section{Die Teilbarkeit der Einwilligungs- erklärung \\ $\nabla$}

Grundsätzlich sind die Gedanken des OLG Braunschweig nachvollziehbar und wichtig, erscheinen angesichts der konkreten Formulierung der streitgegenständlichen Einwilligungserklärung jedoch etwas gekünstelt. In der Einwilligungserklärung war die mögliche Abtretung an die Bank in so unmittelbarem Zusammenhang mit der ersten Abtretung an die Abtretungsstelle behandelt worden, dass eine Einwilligung des Patienten in die Datenweitergabe auch an die Bank naheliegt. Der BGH beschäftigte sich jedoch mit der Frage der wirksamen Einwilligung in die Abtretung an die Bank nicht, da diese im Sachverhalt tatsächlich gar nicht vorgenommen wurde und daher gar nicht darüber zu entscheiden sei. Die Karlsruher Richter werteten allerdings im Gegensatz zum OLG Braunschweig die Abtretung an die Abrechnungsstelle als wirksam. Sie begründeten dies damit, dass die einzelnen Punkte der verwendeten Einwilligungserklärung allgemeine Geschäftsbedingungen (AGB) im Sinne der $\S \S 305$ ff. BGB darstellten. Beim Vorliegen von AGB könne der Vertrag nach $\S 306$ Abs. 1 BGB grundsätzlich wirksam bleiben, auch wenn man die Unwirksamkeit einzelner Klauseln unterstellte. Selbst bei einem sprachlichen $\mathrm{Zu}$ sammenhang zwischen einzelnen Klauseln liegt nach der Rechtsprechung des BGH nämlich nur dann eine Gesamtnichtigkeit des Vertrages vor, wenn der noch übrig bleibende wirksame Teil nicht mehr als sinnvoll erscheint und der unwirksame Klauselteil so bedeutend ist, dass mit ihm der bisherige Vertrag steht und fällt. Daher muss die unwirksame Regelung zunächst gedanklich gestrichen werden und dann beurteilt werden, ob der Vertrag auch ohne sie Sinn macht. Diese Vorgehensweise des Streichvorgangs und die entsprechende Überprüfung werden in der Rechtswissenschaft als „blue-penciltest" bezeichnet.

Der BGH hält nach entsprechender Prüfung das Einverständnis in die Schweigepflichtentbindung in persönlicher, zeitlicher und sachlicher Hinsicht für teilbar. Diese Teilbarkeit wirke auch bei der im Sachverhalt vorliegenden Abtretungskonstruktion. Die Abtretung an die Abrechnungsgesellschaft und die Folgeabtretung an die Bank hängen nach Ansicht des BGH nicht so eng zusammen, dass die erste $A b$ tretung zur Abrechnung unsinnig wäre, da die Abrechnungsgesellschaft die Forderungen im eigenen Namen einziehen und gegebenenfalls gerichtlich durchsetzen könne. Eine neue und abweichende Vertragsgestaltung liege nicht vor, zumal die Weiterabtretung an die Bank nicht in jedem Fall erfolge. Die einzelnen Bestandteile der Erklärung können somit auch dann rechtliche Wirkungen entfalten, wenn andere Teile möglicherweise mit Unwirksamkeitsgründen behaftet sind. Die Abtretung an die Abrechnungsstelle war somit wirksam.

\section{Konsequenzen für die Praxis $\nabla$}

Die Konsequenzen aus diesem Urteil sind aus dem Blickwinkel des abtretenden Arztes zunächst günstig. Zum Forderungseinzug und zur gerichtlichen Geltendmachung gegenüber zahlungsunwilligen $\mathrm{Pa}-$ tienten durch eine Abrechnungsstelle kommt es nur auf die Korrektheit der Einwilligung zur „ersten“ Abtretung an. Allerdings muss beachtet werden, dass der BGH als Konsequenz seiner Auslegung nicht die grundsätzlichen Bedenken ausräumen konnte, die das OLG Braunschweig hinsichtlich der ungenügenden Einwilligungserklärung in die Refinanzierungsabtretung geäußert hat. Daher sollte im Praxisformular die Einwilligung in die Refinanzierungsabtretung ebenso deutlich und detailliert formuliert werden wie die Einwilligung in die Abtretung an die Abrechnungsgesellschaft. Dahingehend müssen die verwendeten Formulare in den Arztpraxen und Krankenhäusern überprüft werden. 


\section{Ausblick in die Zukunft}

$\nabla$

Die Fragen hinsichtlich Einwilligung, Schweigepflicht und Datenschutz stellen sich jedoch nicht nur bei Abrechnungen, sondern auch bei anderen modernen Hilfen im Praxisbetrieb. Hierbei sind beispielsweise der Einsatz von Callcentern oder von Datenclouds zu nennen. Vieles ist dabei rechtlich noch ungeklärt und nicht gesetzlich geregelt und wird in der Rechtswissenschaft intensiv diskutiert (vgl. dazu Bucher, MedR 2013, S. 337 ff, 339ff.). Wenn ein Einsatz solcher Mittel angedacht ist, sollte daher in jedem Fall Expertenrat eingeholt werden.

Lic. iur. can. Urs Fabian Frigger

Rechtsanwalt

Rechtsanwälte Wigge

Scharnhorststraße 40

48151 Münster

www.ra-wigge.de

E-Mail: muenster@ra-wigge.de 